\title{
Inflammasome activity in leucocytes decreases with abdominal aortic aneurysm progression
}

\author{
PHILIPP ERHART ${ }^{1}$, SINAN CAKMAK $^{1}$, CASPAR GROND-GINSBACH $^{2}$, \\ MAANI HAKIMI $^{1,3}$, DITTMAR BÖCKLER ${ }^{1,3}$ and SUSANNE DIHLMANN ${ }^{1,3}$ \\ ${ }^{1}$ Department of Vascular and Endovascular Surgery and ${ }^{2}$ Neurology Department, \\ University Hospital Heidelberg; ${ }^{3}$ Vascular Biobank Heidelberg, D-69120 Heidelberg, Germany
}

Received April 4, 2019; Accepted July 19, 2019

DOI: 10.3892/ijmm.2019.4307

\begin{abstract}
Abdominal aortic aneurysms (AAAs) are characterized by chronic inflammatory cell infiltration. The present extended immunohistochemistry study aimed to characterize inflammation in AAA and aortic control samples. In specific, the composition of the infiltrating immune cells and the expression of five inflammasome components in these immune cells were evaluated, in order to characterize their role in AAA development. A total of 104 biopsies from 48 AAA patients and 40 healthy specimens from organ donors were evaluated for their grade of inflammation. Infiltrating leukocytes were characterized by specific markers (CD3, CD20 and CD68), intramural localization and inflammasome protein expression [NLR family pyrin domain containing 3 (NLRP3), absent in melanoma 2 (AIM2), apoptosis-associated speck-like protein containing a caspase recruitment domain (ASC), Caspase-1 and Caspase-5]. Macrophages, B and T lymphocytes were detected to a similar extent in grade 1, 2 and 3 AAA specimens, whereas in control samples, B and $\mathrm{T}$ lymphocytes were rarely observed in grade 1 lesions. Expression frequencies of NLRP3, AIM2 and Caspase-5 were significantly higher in grade 1 lesions of AAA samples compared with grade 1 lesions in control samples. Finally, AIM2, ASC, and Caspase-5 displayed significantly lower expression frequencies in grade 3 compared with grade 2 AAA specimens, and all inflammasome components were less frequently detected in grade 3 than in grade 1 lesions of AAA. This indicates that inflammasome activities decrease with AAA progression in infiltrating leukocytes. No statistically significant association was found for grade 2 and grade 3 lesions and total leukocyte count, C-reactive protein levels, maximal aortic diameter, plasma
\end{abstract}

Correspondence to: Dr Philipp Erhart, Department of Vascular and Endovascular Surgery, University Hospital Heidelberg, Im Neuenheimer Feld 110, D-69120 Heidelberg, Germany E-mail: philipp.erhart@med.uni-heidelberg.de

Key words: Caspase-1, Caspase-5, inflammation, absent in melanoma 2, NLR family pyrin domain containing 3, abdominal aortic aneurysm, finite element analysis cholesterol level or biomechanical parameters (derived from finite element analysis) of the respective patients. Overall, the aortic wall of AAA contained lymphocytes and macrophages with different states of activity. The present data suggested that therapeutic inhibition of specific inflammasome components might counteract AAA development and progression.

\section{Introduction}

Abdominal aortic aneurysm (AAA), a progressive pathological dilatation of the aortic wall, occurs mostly asymptomatic, but may become life threatening in case of rupture. The pathophysiology of AAA is a complex multifactorial process that remains incompletely understood. Common risk factors for this disease are male gender, age, smoking and hypertension $(1,2)$. In addition, familial clustering points to inheritance of predisposing genetic alterations $(3,4)$. Histologically, AAA is characterized by chronic inflammation, loss of vascular smooth muscle cells, and degradation of elastic fibers and extracellular matrix (ECM) $(5,6)$. For many years, atherosclerosis was considered a dominant cause for AAA progression. However, increasing evidence suggests that inflammatory reactions and autoimmunity across the tunica media/adventitia junction are important factors for aortic destruction and dilatation $(7,8)$. Inflammatory infiltrates were described even 30 years ago in the adventitial layer of AAA without any clinical signs of inflammation (9). A few years later, an immune-mediated response was suggested, after characterization of the inflammatory cells present in the aortic wall of AAA specimens (10). Since that time, many others have confirmed the presence of $\mathrm{T}$ and $\mathrm{B}$ lymphocytes, macrophages, mast cells and neutrophils (11-15). The presence of $\mathrm{B}$ and $\mathrm{T}$ lymphocytes points to an antibody-mediated humoral immunity and potentially an autoimmune response driving AAA progression $(14,15)$. In addition, the different inflammatory/immune cells release proinflammatory cytokines, including tumor necrosis factor (TNF)- $\alpha$, interleukin (IL)-6, IL-1 $\beta$ and interferon (IFN)- $\gamma$, all of which have been detected in the wall of AAA specimens (16).

Regardless of the increasing knowledge on inflammatory cells and mediators, the initial trigger for inflammation and the precise order of the process, resulting in a destructive autoimmune response to the aortic wall, is unknown. A very early event in response to danger signals on cells is 
activation of inflammasomes, sensing many environmental and pathogen/host-derived factors $(17,18)$. The inflammasomes are a family of cytoplasmic multiprotein complexes required for maturation of the IL-1 $\beta$ and IL-18 cytokines, which are key regulators of immune response and tissue homoeostasis (19). Because deregulated activity of IL-1 is linked to autoimmune and inflammatory diseases (20), inflammasomes are tightly regulated at both the transcriptional and protein level. Depending on the initial sensor, several subfamilies are distinguished: The NOD-like receptors (NLR), such as NLR family pyrin domain containing 3 (NLRP3), act as sensors for intracellular damage-associated signals, including cholesterol crystals, nanoparticles and reactive oxygen species. A second subfamily, that includes absent in melanoma 2 (AIM2), acts as sensors for intracellular dsDNA (17). Upon sensing of the danger signal, both subfamilies induce assembly of a multiprotein complex consisting of the apoptosis-associated speck-like protein containing a caspase recruitment domain (ASC) and one of the inflammatory caspases, Caspase-1 or Caspase-5. In the present study, therefore, NLRP3, AIM2, ASC, Caspase-1 and Caspase-5 were defined as relevant inflammasome markers for investigation. Although initially detected in monocytes, it is now evident that inflammasomes are expressed and assembled in response to different stimuli in many myeloid and non-myeloid cell types, including epithelial, mesenchymal and neuronal cells $(21,22)$.

In a recent pilot study, our group demonstrated high levels of inflammasome components in lymphocytic infiltrates of AAA (23). The present study aimed to characterize inflammation in different AAA stages in more detail, according to the composition of the infiltrating immune cells and according to the expression of NLRP3, AIM2 and ASC in these immune cells. Additionally, the hypothesis that the inflammasome activity may differ between different stages of AAA progression was examined.

\section{Materials and methods}

Tissue sampling and patient selection. Formalin-fixed and paraffin-embedded (FFPE) biopsies derived from AAA patients were provided by the Vascular Biobank Heidelberg (VBBH). A total of 104 aortic wall specimens from 48 patients (AAA group) were collected during open surgery between February 2013 and April 2014 at the Department of Vascular and Endovascular Surgery, Heidelberg. Tissue sampling from these patients was based on preoperative finite element analysis (FE-A), identification of AAA wall regions with highest and lowest peak wall rupture index (PWRI, see below) and extraction according to clockwise orientation from FE-A measurement protocols. In addition, 40 aortic- and visceral artery samples (control group), free of macroscopic disease, were obtained during organ transplantations from anonymous individuals. All patients gave their written informed consent to the study and all samples were processed immediately and stored in the VBBH for further histological and immunohistochemical analysis.

Patient characteristics. Laboratory parameters [C-reactive protein (CRP) plasma level,leukocyte count, cholesterol plasma level] and patient specific characteristics (smoking history, diabetes type II medication, hypercholesterinemia, hypertension, and statin therapy) of the AAA group were collected from the patient information system of the University Hospital Heidelberg (ISH-MED). No clinical or personal data were available from the control group. The patient characteristics of the AAA group are summarized in Table I.

$F E-A$. Preoperative FE-A was performed for every patient of the AAA group by a single investigator using the commercially available A4-Clinics software (VASCOPS GmbH). For analysis, Digital Imaging and Communications in Medicine (DICOM) data were used from computer tomography angiography (CT-A; in plane solution $0.33 \mathrm{~mm}$, slice thickness $0.7-3.3 \mathrm{~mm}$ ) to detect AAA wall regions between the renal arteries and aortic bifurcation. Semiautomatic FE-A calculation is based on the subsequent steps of luminal and exterior AAA surface recognition, three dimensional mesh generation and computation of biomechanical parameters. The later step incorporates patient specific (blood pressure, sex, smoking history) and anatomical boundary conditions (thrombus, vessel morphology) for calculating peak wall stress (PWS; in $\mathrm{kPascal}$ ), peak wall rupture risk index (PWRI) and intraluminal thrombus size (ILT; in $\mathrm{cm}^{3}$ ). The three dimensional analysis report, marking regions with highest and lowest PWRI values, was used to extract samples during open surgery based on clockwise orientation. FE-A data included morphological (AAA diameter, thrombus volume) and biomechanical parameters (PWS and PWRI).

Immunohistochemistry. Processing and immunohistochemical staining was performed following standard procedures by using the following primary antibodies: Mouse-anti-human CD45 (1:1,000; \#3575, pan-leukocyte marker; Cell Signaling Technology,Inc.),rabbit anti-human CD3 (1:400; \#85061, T-cell marker; Cell Signaling Technology, Inc.), rabbit anti-human NLRP3 (1:500; \#13158; Cell Signaling Technology, Inc.), rabbit anti-human Caspase-1 (1:50; \#85061; Cell Signaling Technology, Inc.), mouse-anti-human CD68 (1:2,000; \#M0876, macrophage marker; Dako; Agilent Technologies, Inc.), rabbit anti-human AIM2 (1:200; \#HPA031365; Sigma-Aldrich; Merck KGaA), rabbit anti-human ASC (\#ADI-905-173; Enzo Life Sciences, Inc.), and rabbit anti-human Caspase-5 (1:200; \#3029, BioVision, Inc.). Detailed staining protocols are available from the authors upon request. Briefly, $4 \mu \mathrm{m}$ sections were deparaffinized, rehydrated and incubated in $100 \mathrm{mM}$ citrate buffer $\mathrm{pH} 6.0$ for antigen retrieval, prior to incubation with the primary antibody at $4^{\circ} \mathrm{C}$ overnight. After washing, detection was performed by using the Dako REAL Detection System Peroxidase/AEC rabbit/mouse (Dako; Agilent Technologies, Inc.), according to the recommendations of the manufacturer. All sections were counterstained with hematoxylin. Immunohistochemical staining for the B-cell marker CD20 was performed by the Institute of Pathology of the University Hospital Heidelberg, according to standard diagnostic procedures.

Grading of inflammatory lesions in aortic samples. Histopathological grading of inflammatory lesions and aortic samples was performed based on the intramural location of $\mathrm{CD} 45^{+}$cells. Briefly, grade 0 describes a healthy vessel wall 
Table I. Patient characteristics of the AAA cohort.

\begin{tabular}{lc}
\hline Parameter & AAA $(\mathrm{n}=46)$ \\
\hline Mean age, years (range) & $62.2(47-87)$ \\
Male, n (\%) & $40(87.6)$ \\
Current smoker, n (\%) & $24(52.2)$ \\
Non-smoker, n (\%) & $9(19.6)$ \\
Medication for & \\
Type 2 diabetes, n (\%) & $4(10.4)$ \\
Hypercholesterinemia, n (\%) & $39(87.2)$ \\
Hypertension, n $(\%)$ & $42(91.7)$ \\
\hline
\end{tabular}

without inflammation and few, isolated leukocyte infiltrates. A Grade 1 lesion describes a mild chronic inflammation with leukocyte infiltrations located to atherosclerotic plaques. A Grade 2 lesion describes a moderate chronic inflammation with localized and diffuse lymphocyte infiltrates within the tunica adventitia. A Grade 3 lesion refers to a severe chronic vessel wall inflammation with lobular arrangement of lymphocyte infiltrates at the media-adventitia border. Examples of the grades are presented in Fig. 1. Two to three samples from each aneurysm were used for analysis and the highest lesion grade of inflammation in the sample of each patient was used to categorize the total inflammation grade of this aneurysm (patient sample).

Statistical analysis. Only informative samples, i.e. sections showing the lesion of interest with a technically reliable staining quality, were used for statistical analysis. Therefore, the total number of samples included in the statistical analysis was sometimes lower than the total number of AAA or control tissues. Immunohistochemically identified differences in the grade of inflammation between AAA and control patient groups, as well as differences in expression of inflammasome components between grades and between AAA and control groups, were tested for significance by Barnard's unconditional test for $2 \times 2$ contingency tables (24), using CRAN-R package version 1.8 (https://github.com/kerguler/Barnard). Global heterogeneity of inflammasome component expression across the grades of inflammation (I-III) was calculated with the Fisher exact $2 \times 3$ test. Patient characteristics and FEA data were compared with histopathological findings (inflammation grading and inflammasome component expression) using the same test. For the comparison of the histological data with the clinical features of the patients, student's t-test was applied using SPSS (version 25; IBM Corp.).

\section{Results}

Categorization of samples according to the $\mathrm{CD}^{4} 5^{+}$cell infiltration pattern. To categorize the inflammation grade and to locate intramural inflammatory cells in AAA and control tissues, FFPE sections of all samples were initially analyzed for CD45 expression by immunohistochemistry. In agreement with our previous pilot study (23), all examined AAA specimens exhibited visible signs of vessel wall inflammation.
None of the 46 AAA samples was categorized as grade 0 or grade 1, 28/46 (60.8\%) were categorized as grade 2 and 18/46 (39.1\%) as grade 3 (Table II and Fig. 1). By contrast, none of the 40 control samples exhibited profound inflammatory cell infiltration (grade 3) and only 3/40 samples were categorized grade 2 (Table II and Fig. 1). Because samples categorized as grade 3 (lobular arrangement of CD $45^{+}$cells) did additionally show grade 2 and 1 infiltrates, and samples categorized as grade 2 (diffuse infiltration) additionally displayed grade 1 lesions (isolated $\mathrm{CD} 45^{+}$cells located to atherosclerotic plaque), the number of individual lesions was higher than the total number of samples (Table III).

The pattern of inflammatory cell phenotypes within the same inflammation grade differs between AAA samples and controls. Next, the composition of different leukocyte phenotypes in each inflammation grade of healthy and AAA tissue samples was analyzed. Macrophages (CD68 ${ }^{+}$cells), B lymphocytes (CD20 ${ }^{+}$cells) and T cells (CD3 ${ }^{+}$cells) have been identified in AAA for decades (14). The present study aimed to compare the leukocyte pattern in the different inflammatory lesions and total inflammation grades between control and AAA tissue, as identified in Table II.

Macrophages $\left(\mathrm{CD}^{+} 8^{+}\right.$cells $)$were detected in a similar frequency in grade 1 lesions of both AAA and control samples [43/44 (97.7\%) vs. 20/21 (95.2\%), respectively, of informative samples], and were predominantly located around the atherosclerotic plaque (Fig. 2A and J). By contrast, grade 1 lesions of AAA samples were significantly more infiltrated with $\mathrm{T}$ lymphocytes (CD3 ${ }^{+}$cells) than control samples [38/46 (82.6\%) vs. 5/21 (23.8\%), respectively; $\mathrm{P}<0.001$; Fig. $2 \mathrm{~B}$ and $\mathrm{K}]$. Likewise, B lymphocytes (CD20+ cells) were more frequent in grade 1 lesions of AAA samples than in control samples [32/46 (69.6\%) vs. $2 / 21$ (9.5\%); P<0.001; Fig. 2C and L].

In grade 2 lesions (diffusely clustered leukocytes in the tunica media and adventitia), macrophages were detected at similar frequencies in both AAA samples and controls [41/43 $(95,4 \%)$ vs. $2 / 3$ (66.7\%), respectively; $\mathrm{P}=0.093$; Fig. $2 \mathrm{D}$ and $\mathrm{J}]$. In addition, AAA and control samples were equally infiltrated with T lymphocytes (100\% of samples; Fig. 2E and K). By contrast, B lymphocytes were exclusively found in grade 2 lesions of AAA samples, and not in controls [44/45 (97.8\%) vs. 0/3 (0.0\%), respectively; $\mathrm{P}<0.001$; Fig. $2 \mathrm{~F}$ and L].

Grade 3 lesions (lobular accumulations of leukocytes) were exclusively detected in AAA samples (Fig. 2G and L) and heavily infiltrated with macrophages [13/16 (81.3\%)], T lymphocytes [14/14 (100\%)] and B lymphocytes [14/15 $(93.3 \%)]$.

The inflammasome expression pattern within the same inflammation grade differs between AAA samples and controls. To refine the findings of our previous pilot study (23), the present study compared next the frequencies of inflammasome components in each lesion grade between AAA and control samples. In AAA samples, grade 1-associated expression of NLRP3, AIM2, ASC and Caspase-1 was detected in 100\% of samples, whereas $80.4 \%$ of AAA samples were positive for Caspase-5 expression (Fig. 3A-E and P-T). Similarly, ASC and Caspase-1 were expressed in $100 \%$ of grade 1 lesions in control samples (Fig. 3R and S). The expression frequencies of NLRP3 and 


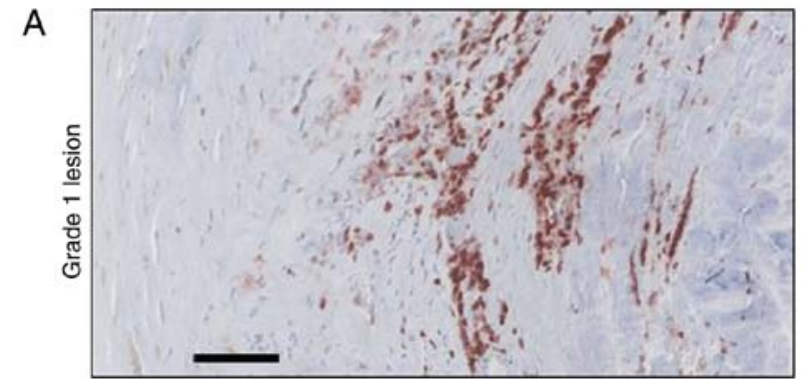

B

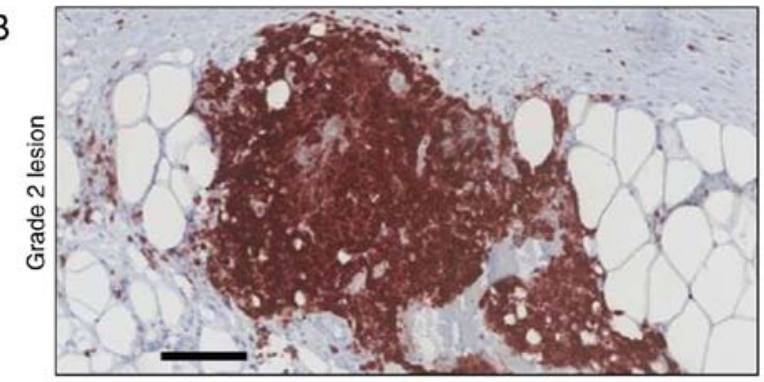

C

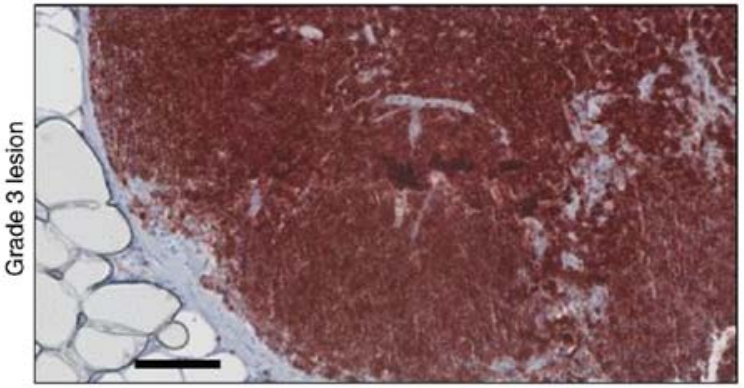

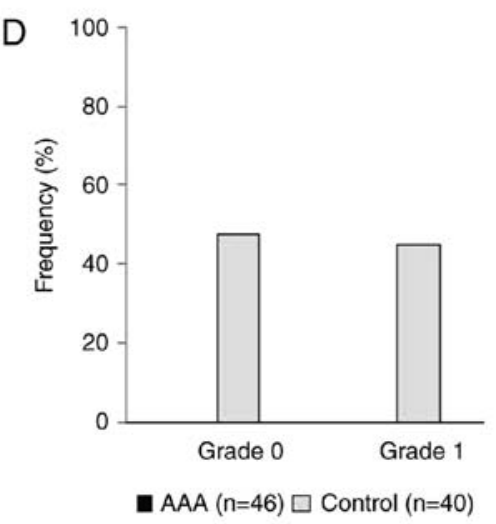

E

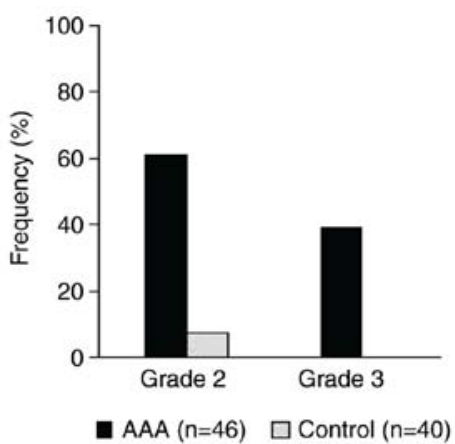

Figure 1. Grading of AAA wall inflammation. Representative examples of (A) grade 1, (B) grade 2 and (C) grade 3 lesions in the AAA wall. Sections from formalin-fixed and paraffin-embedded biopsies were immunohistochemically labelled with anti-CD45 antibody to visualize leukocytes of different phenotypes (red). Scale bar, $100 \mu \mathrm{m}$. (D) Frequencies of grade 0 and grade 1 lesions in AAA and control samples are presented as percentages. (E) Frequencies of grade 2 and 3 lesions in AAA and control samples are presented as percentages. AAA, abdominal aortic aneurysm.

AIM2 were significantly lower in grade 1 lesions of control samples [57.1\% (12/21), $\mathrm{P}<0.001$ and $17 / 21$ (81.0\%), $\mathrm{P}=0.008$, respectively; Fig. 3P and Q].

The grade 2-associated expression frequency of NLRP3 and AIM2 appeared higher in AAA samples compared with control samples $[59.9 \%(26 / 44)$ vs. $0 \%(0 / 3), \mathrm{P}=0.082$; and $90.7 \%$ (39/43) vs. $66.7 \%$ (2/3), $\mathrm{P}=0.298$, respectively; Fig. 3P and Q], although these differences were not statistically significant, most likely due to the low amount of grade 2 lesions in control samples. ASC, Caspase-1 and Caspase-5 expression frequencies did not differ between the two groups $[100 \%$ (44/44) vs. $100 \%(3 / 3), P=1 ; 97.8 \%$ (45/46) vs. $100 \%(2 / 2)$, $\mathrm{P}=1$; and $60.9 \%(28 / 46)$ vs. $100 \%$ (3/3), $\mathrm{P}=0.239$, respectively; Fig. 3R-T].

In grade 3 lesions that were exclusively detected in AAA samples, NLRP3 was expressed in 6/13, AIM2 in 9/15, ASC in 11/14, Caspase-1 in 15/16 and Caspase-5 in 3/15 informative samples.

Inflammasome expression decreases from grade 1 to grade 3 lesions in human AAA tissues. For testing of heterogeneity of inflammasome component expression across different inflammation grades (I-III) the Fisher test was used. Based on our previous pilot study, the present study hypothesized that the expression of inflammasome components might change during
AAA progression. As presented in Fig. 4, the frequencies of all inflammasome components, except Caspase-1, were significantly different across the inflammation grades (NLRP3, $\mathrm{P}<0.001$; AIM2, $\mathrm{P}<0.001$; ASC, $\mathrm{P}=0.002$; Caspase-1, $\mathrm{P}=0.148$; and Caspase-5, $\mathrm{P}<0.001)$. These results suggested a different innate immune response in inflammatory regions around atherosclerotic areas compared with the immune response at the media/adventitia border. In addition, AIM2, ASC and Caspase-5 expressions indicated a gradual decrease of inflammasome positive leukocytes during AAA progression (Fig. 4). An overall comparison of expression frequencies of leukocyte cell markers and inflammasome proteins for AAA and control samples is detailed in Table IV.

The immune response within the AAA tissue is not associated with plasma inflammatory markers, the maximal $A A A$ diameter or predicted rupture risk. Using FE-A, our group has previously analyzed whether histological features of AAA correlate with predicted rupture risk and clinical parameters of AAA patients $(25,26)$. To investigate, whether the intramural immune response analyzed in the present study may be reflected by any diagnostic marker that is clinically used for AAA disease control, different parameters were tested for association. Neither clinical inflammatory characteristics (CRP levels, leukocyte count and plasma cholesterol levels), 
Table II. Categorization of samples according to the $\mathrm{CD} 45^{+}$cell infiltration pattern.

\begin{tabular}{lcccc}
\hline Sample & Grade 0,n $(\%)$ & Grade 1, n $(\%)$ & Grade 2, n (\%) & Grade 3, n (\%) \\
\hline AAA $(\mathrm{n}=46)$ & $0(0.0)$ & $0(0.0)$ & $28(60.8)$ & $18(39.1)$ \\
Control $(\mathrm{n}=40)$ & $19(47.5)$ & $18(45)$ & $3(7.5)$ & $0(0.0)$ \\
\hline
\end{tabular}

$\mathrm{n}$, number of patients (sample size); AAA, abdominal aortic aneurysm group.

Table III. Grading of individual lesions within the samples according to the $\mathrm{CD} 45^{+}$cell infiltration pattern.

\begin{tabular}{lcccc}
\hline Sample & $\begin{array}{c}\text { Grade 0 lesions } \\
\text { n/total samples }\end{array}$ & $\begin{array}{c}\text { Grade 1 lesions } \\
\text { n/total samples }\end{array}$ & $\begin{array}{c}\text { Grade 2 lesions } \\
\text { n/total samples }\end{array}$ & $\begin{array}{c}\text { Grade 3 lesions } \\
\text { n/total samples }\end{array}$ \\
\hline AAA & $0 / 46$ & $46 / 46$ & $44 / 46$ & $18 / 46$ \\
Control & $19 / 40$ & $21 / 40$ & $3 / 40$ & $0 / 40$ \\
\hline
\end{tabular}

$\mathrm{n}$, number of patients (sample size); AAA, abdominal aortic aneurysm group.
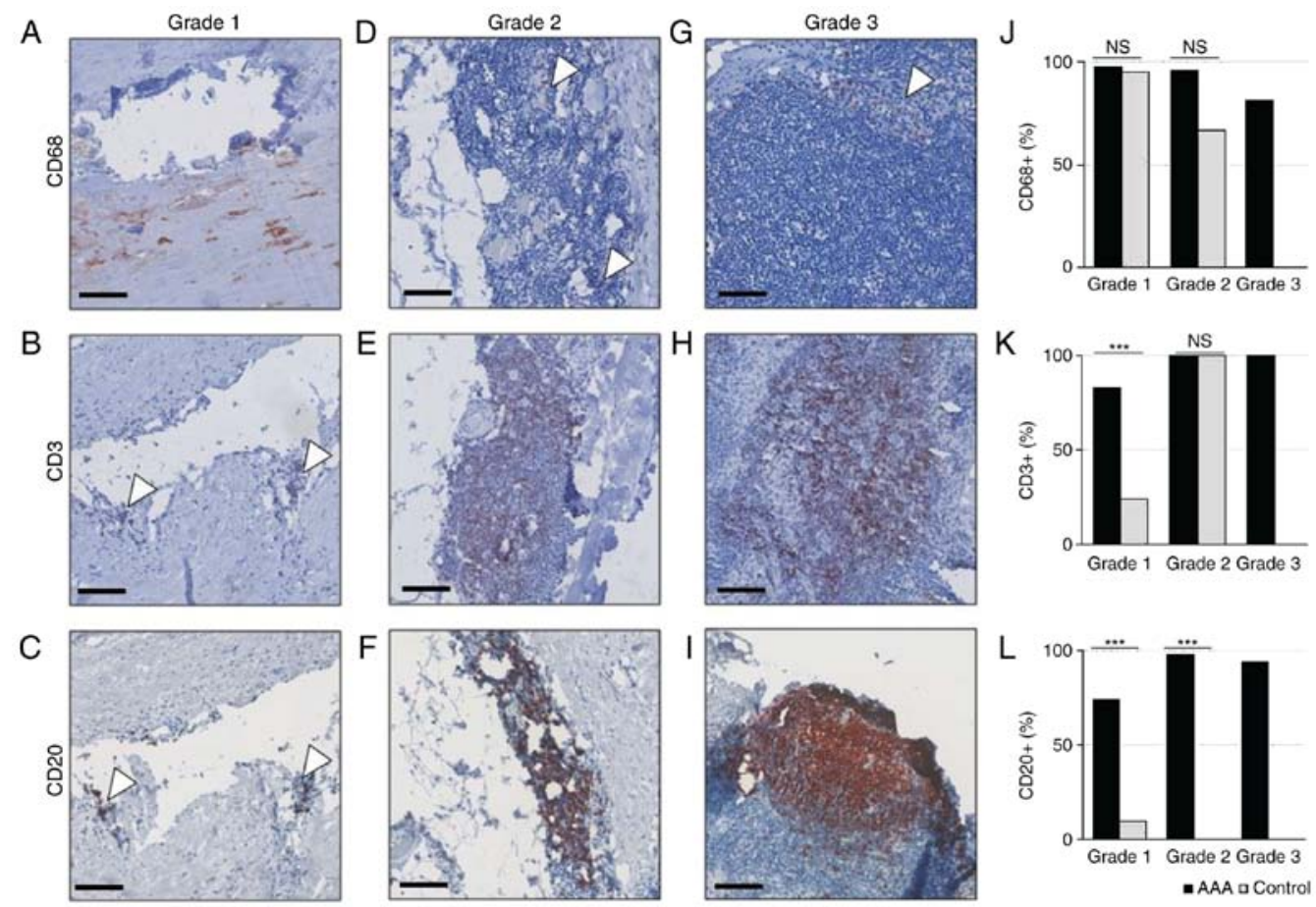

Figure 2. Immunohistochemical analysis of inflammatory infiltrates in AAA and healthy controls. (A-C) Grade 1, (D-F) grade 2 and (G-I) grade 3 lesions were stained with anti-CD68 to detect macrophages, with anti-CD3 to detect T lymphocytes, or with anti-CD20 to detect B- lymphocytes, as indicated. Scale bar, $100 \mu \mathrm{m}$. (J) Distribution of CD68 ${ }^{+}$cells in grade 1, 2 and 3 lesions of AAA and control samples. (K) Distribution of CD3 ${ }^{+}$cells in grade 1,2 and 3 lesions of AAA and control samples. (L) Distribution of CD20 cells in grade 1,2 and 3 lesions of AA and control samples. Arrows point to positive isolated cell clusters. ${ }^{* * * *} \mathrm{P}<0.001$, comparisons are indicated by lines (Barnard's test). AAA, abdominal aortic aneurysm; ns, not significant.

nor parameters from FE-A (PWS, PWRR and maximal diameter), were associated with the respective inflammatory grade, predominant leukocyte phenotype or inflammasome protein expressions within individual lesions (data not shown). Thus, a higher grade of inflammation within the AAA group was not necessarily associated to a higher systemic inflammatory response (CRP, leucocyte count) or AAA vessel wall regions with a higher calculated rupture risk from FE-A.

\section{Discussion}

Emerging evidence suggests that chronic intramural inflammation, involving a variety of inflammatory cell types, is the major driving force of AAA progression $(14,27)$. The presence of $\mathrm{T}$ and $\mathrm{B}$ lymphocytes argues for autoimmunity as an etiological component of AAA pathophysiology, which involves both adaptive and innate immunity (7). Given that 

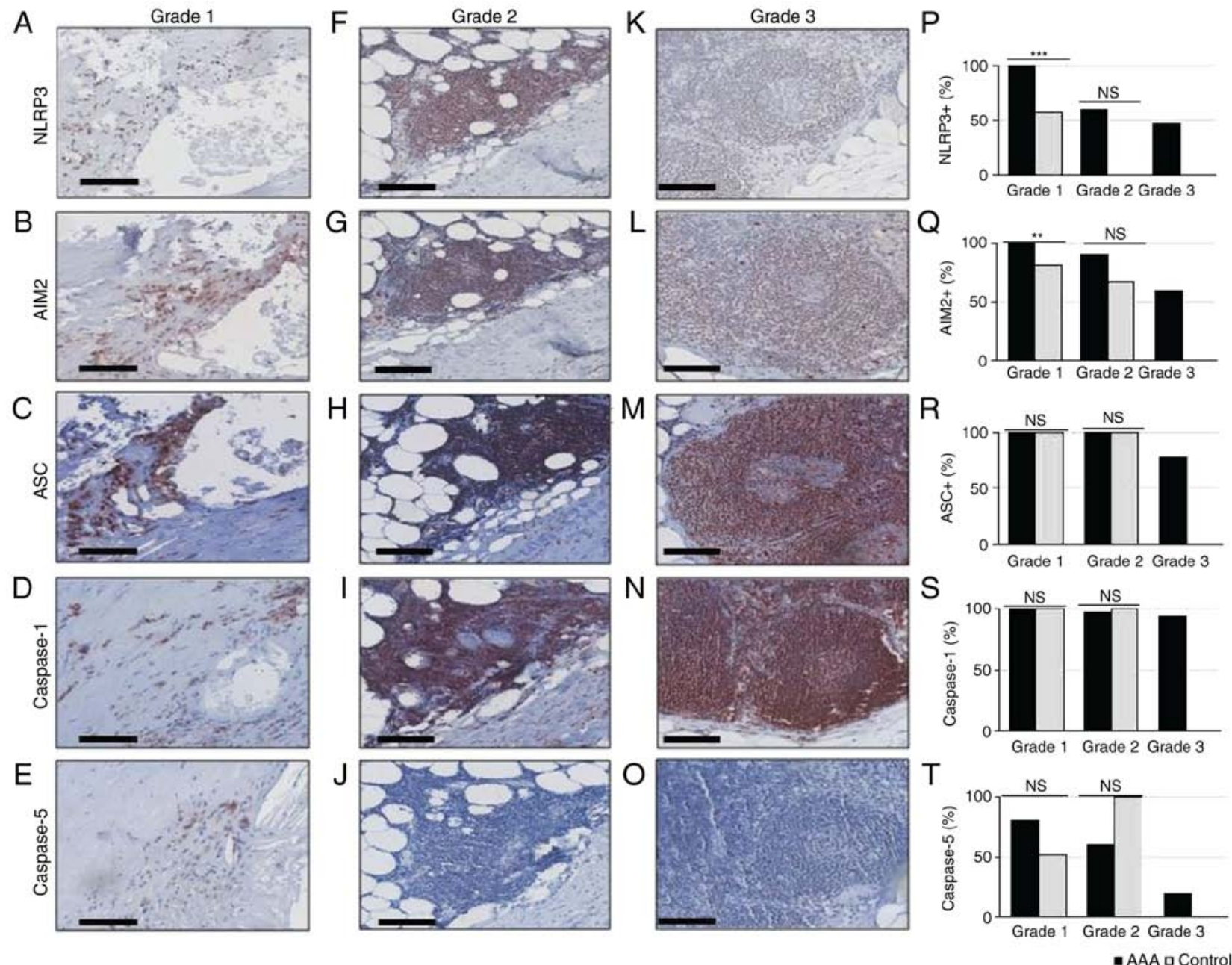

Figure 3. Immunohistochemical analysis of inflammatory infiltrates in AAA and healthy controls. (A-E) Grade 1, (F-J) grade 2 and (K-O) grade 3 lesions were stained with anti-NLRP3, anti-AIM2, anti-ASC, anti-Caspase-1 or anti-Caspase-5, as indicated. Scale bar, $100 \mu \mathrm{m}$. (P) Distribution of NLRP3 ${ }^{+}$cells, (Q) $\mathrm{AIM}^{+}$cells, (R) ASC ${ }^{+}$cells, (S) Caspase $-1^{+}$cells, and (T) Caspase $-5^{+}$cells in grade 1,2 and 3 lesions of AAA and control samples. ${ }^{* *} \mathrm{P}<0.01$ and ${ }^{* * *} \mathrm{P}<0.001$, comparisons are indicated by lines (Barnard's test). AAA, abdominal aortic aneurysm; NLRP3, NLR family pyrin domain containing 3; AIM2, absent in melanoma 2; ASC, apoptosis-associated speck-like protein containing a caspase recruitment domain; ns, not significant.

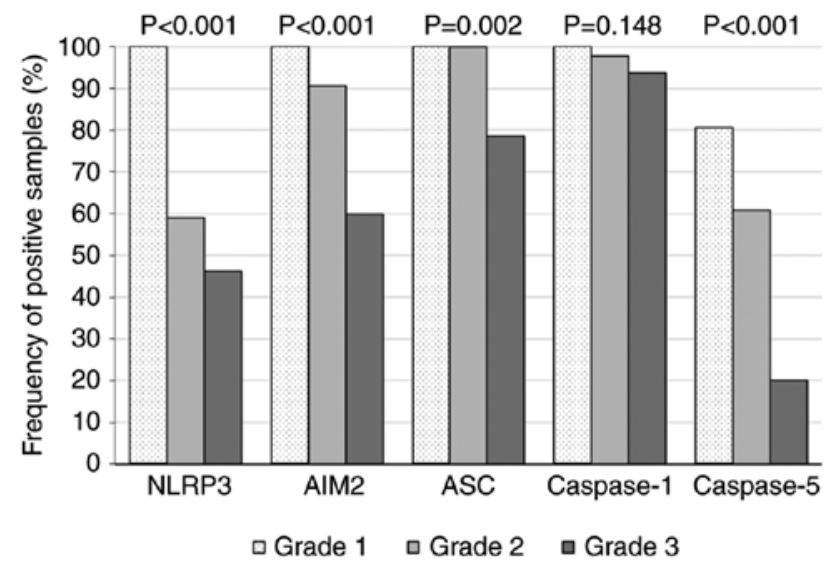

Figure 4. Inflammasome-related protein expression frequencies decrease with AAA progression. Distribution of NLRP3, AIM2, ASC, Caspase-1 and Caspase-5 expression frequencies were determined by immunohistochemistry as described in Fig. 3. For statistical analysis, the Fisher exact test $(2 \times 3$ table) was used to detect heterogeneity across groups. For statistical calculations, the numbers listed in Table IV were used. Significant P-values indicate heterogeneity across the three groups (null hypothesis states that all groups are equal). AAA, abdominal aortic aneurysm; NLRP3, NLR family pyrin domain containing 3; AIM2, absent in melanoma 2; ASC, apoptosis-associated speck-like protein containing a caspase recruitment domain. activation of inflammasomes in hematopoietic cells is an initiating step in innate immunity, and sterile inflammation is implicated in cardiovascular diseases $(28,29)$, the present study addressed the leukocyte composition and the inflammasome expression pattern in different inflammatory grades of AAA compared with control (apparently healthy) tissues. The results demonstrated that: i) Whereas in control samples, grade 1 (atherosclerotic) lesions were predominantly infiltrated with macrophages, the majority of AAA grade 1 lesions were additionally infiltrated with $\mathrm{B}$ and T lymphocytes; ii) The expression frequencies of the inflammasome components NLRP3, AIM2 and Caspase-5 were significantly higher in grade 1 lesions of AAA samples compared with grade 1 lesions in control samples; and iii) AIM2, ASC, and Caspase-5 displayed significantly lower expression frequencies in grade 3 compared with grade 2 AAA specimens, and all inflammasome components were less frequently detected in grade 3 compared with grade 1 lesions of AAA.

The detection of different infiltrating cell types in histologically similar areas (grade 1 lesion, atherosclerotic plaque) between AAA and non-AAA samples suggests different 


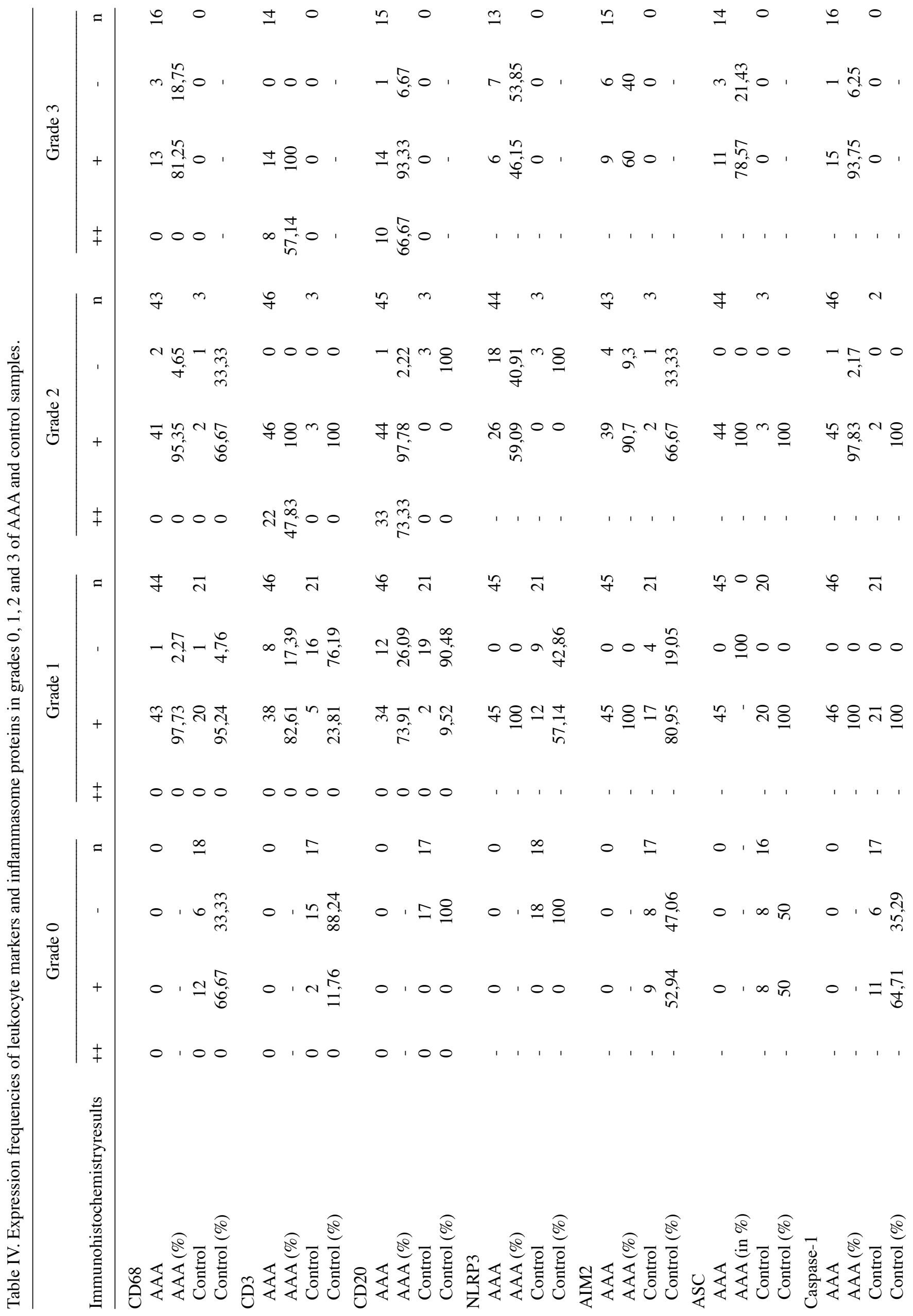


pathological mechanisms. B and T lymphocytes do not only infiltrate the adventitial layer of AAA, as described previously $(10,30,31)$, but are also frequent in atherosclerotic areas of AAA, in contrast to atherosclerotic areas of non-AAA arteries. The reasons for this imbalance remain unclear. The present findings support the hypothesis of inflammasome-mediated innate immunity in AAA pathology. The expression patterns of NLRP3, AIM2, ASC, and Caspase-1 overlap particularly with that of $\mathrm{T}$ and $\mathrm{B}$ lymphocytes, suggesting that these cell types, rather than macrophages, display inflammasome activity in AAA tissues. Double-staining by using immunofluorescence on cryosections will help to allocate inflammasome expressions to certain cell types more precisely.

Irrespective of the cell type, the expression of inflammasome components appears to decline during AAA progression, according to the present results. Assuming that well-organized lobular lymphocyte infiltrations (grade 3 lesions) develop later in AAA than diffuse lymphocyte infiltrations (grade 2 lesions) and infiltration of atherosclerotic areas (grade 1 lesions), the decline of inflammasome expression frequencies from grade 1 to grade 3 lesions argues for a change of the immune response during AAA progression. It is thus tempting to speculate that signaling by inflammasomes is an early event of the inflammatory response by lymphocytes, whereas later these lymphocytes perform other mechanisms in AAA pathology. Alternatively, the different inflammation grades occur independently within AAA tissues and represent different etiologies. However, the simultaneous presence of different infiltration grades within the same sample argues for the hypothesis of a progressive change from single cells, over diffuse aggregates, and finally lymphoid follicles with B-cells forming germinative centers.

The role of inflammasomes in AAA has been extensively studied in animal experiments. Genetic deletion of NLRP3, ASC or Caspase-1 protected ApoE-deficient mice from angiotensin II-induced aortic aneurysms (29). The incidence, maximal diameter and severity of AAA, as well as adventitial fibrosis and inflammatory responses, were significantly reduced in inflammasome-deficient mice (29). In addition, genetic and pharmacological disruption of IL-1 $\beta$, the major product of inflammasome activity, has been demonstrated to inhibit experimental aortic aneurysm formation $(32,33)$. In humans, the CANTOS trial, a randomized, double-blind, placebo-controlled trial of canakinumab, an IL- $1 \beta$ neutralizing antibody, in 10,061 patients, was shown to reduce cardiovascular event rates (34-36). This was the first study demonstrating that targeting an inflammasome-activated cytokine can prevent cardiovascular events in high risk patients. Unfortunately, data on AAA development or progression are not available from this trial. The current data, demonstrating inflammasome expression particularly in grade 1 and 2 AAA lesions, supports the hypothesis that therapeutic inhibition of IL- $1 \beta$ or certain inflammasome components might also be effective in attenuating AAA development and progression.

A limitation of the present study was that insufficient clinical information was available for the anonymous organ transplantation patients of the control group. Antidiabetic (glyburide) or antihypertensive medication could influence inflammasome activity, and may thus affect the comparability of the study groups. 
In conclusion, the present findings suggested that different inflammatory areas in the aortic wall of AAA consisted of lymphocytes and macrophages at different states of activity. The current results might help to identify molecular targets for development of therapeutic drugs targeting AAA growth.

\section{Acknowledgements}

We thank Anja Spieler for excellent technical assistance.

\section{Funding}

No funding was received.

\section{Availability of data and materials}

All data generated or analyzed during this study are included in this published article.

\section{Authors' contributions}

PE contributed to the conception and design of the study, data analysis and interpretation, and writing of the manuscript. SC contributed to data analysis and interpretation, data collection, writing and revising the manuscript. CG-G, $\mathrm{MH}$ and DB contributed to data analysis and interpretation, and manuscript revision. SD contributed to data analysis and interpretation, data collection, writing and revising the manuscript. All authors read and approved the final manuscript.

\section{Ethics approval and consent to participate}

Tissue sampling protocol was approved by the Medical Ethics Committee of the University Heidelberg, Germany (reference numbers S-301/2013: Addendum 23.09.2013, 05.07.2016; S-412/2013: Addendum 04.11.2015, 05.01.2016; S-462/2017). All patients gave their written consent prior to the study inclusion.

\section{Patient consent for publication}

Not applicable.

\section{Competing interests}

The authors declare that they have no competing interests.

\section{References}

1. Kent KC, Zwolak RM, Egorova NN, Riles TS, Manganaro A Moskowitz AJ, Gelijns AC and Greco G: Analysis of risk factors for abdominal aortic aneurysm in a cohort of more than 3 million individuals. J Vasc Surg 52: 539-548, 2010.

2. Michel JB, Martin-Ventura JL, Egido J, Sakalihasan N, Treska V, Lindholt J, Allaire E, Thorsteinsdottir U, Cockerill G and Swedenborg J; FAD EU consortium: Novel aspects of the pathogenesis of aneurysms of the abdominal aorta in humans. Cardiovasc Res 90: 18-27, 2011.

3. Golledge $\mathrm{J}$ and Kuivaniemi H: Genetics of abdominal aortic aneurysm. Curr Opin Cardiol 28: 290-296, 2013.

4. Kim HW and Stansfield BK: Genetic and epigenetic regulation of aortic aneurysms. Biomed Res Int 2017: 7268521, 2017.

5. Kuivaniemi H, Ryer EJ, Elmore JR and Tromp G: Understanding the pathogenesis of abdominal aortic aneurysms. Expert Rev Cardiovasc Ther 13: 975-987, 2015.
6. Nordon IM, Hinchliffe RJ, Loftus IM and Thompson MM: Pathophysiology and epidemiology of abdominal aortic aneurysms. Nat Rev Cardiol 8: 92-102, 2011.

7. Jagadesham VP, Scott DJ and Carding SR: Abdominal aortic aneurysms: An autoimmune disease? Trends Mol Med 14: 522-529, 2008.

8. Tilson MD: Decline of the atherogenic theory of the etiology of the abdominal aortic aneurysm and rise of the autoimmune hypothesis. J Vasc Surg 64: 1523-1525, 2016.

9. Beckman EN: Plasma cell infiltrates in atherosclerotic abdominal aortic aneurysms. Am J Clin Pathol 85: 21-24, 1986.

10. Koch AE, Haines GK, Rizzo RJ, Radosevich JA, Pope RM, Robinson PG and Pearce WH: Human abdominal aortic aneurysms. Immunophenotypic analysis suggesting an immune-mediated response. Am J Pathol 137: 1199-1213, 1990.

11. Cohen JR, Keegan L, Sarfati I, Danna D, Ilardi C and Wise L: Neutrophil chemotaxis and neutrophil elastase in the aortic wall in patients with abdominal aortic aneurysms. J Invest Surg 4: 423-430, 1991.

12. Shimizu K, Mitchell RN and Libby P: Inflammation and cellular immune responses in abdominal aortic aneurysms. Arterioscler Thromb Vasc Biol 26: 987-994, 2006.

13. Tsuruda T, Kato J, Hatakeyama K, Kojima K, Yano M, Yano Y, Nakamura K, Nakamura-Uchiyama F, Matsushima Y, Imamura T, et al: Adventitial mast cells contribute to pathogenesis in the progression of abdominal aortic aneurysm. Circ Res 102: 1368-1377, 2008.

14. Dale MA, Ruhlman MK and Baxter BT: Inflammatory cell phenotypes in AAAs: Their role and potential as targets for therapy. Arterioscler Thromb Vasc Biol 35: 1746-1755, 2015.

15. Zhang $\mathrm{L}$ and Wang Y: B lymphocytes in abdominal aortic aneurysms. Atherosclerosis 242: 311-317, 2015.

16. Rizas KD, Ippagunta $\mathrm{N}$ and Tilson MD III: Immune cells and molecular mediators in the pathogenesis of the abdominal aortic aneurysm. Cardiol Rev 17: 201-210, 2009.

17. Latz E, Xiao TS and Stutz A: Activation and regulation of the inflammasomes. Nat Rev Immunol 13: 397-411, 2013.

18. Kono H, Kimura $\mathrm{Y}$ and Latz E: Inflammasome activation in response to dead cells and their metabolites. Curr Opin Immunol 30: 91-98, 2014.

19. Guo H, Callaway JB and Ting JP: Inflammasomes: Mechanism of action, role in disease, and therapeutics. Nat Med 21: 677-687, 2015.

20. Vasanthakumar A and Kallies A: Interleukin (IL)-33 and the IL-1 family of cytokines-regulators of inflammation and tissue homeostasis. Cold Spring Harb Perspect Biol 11: a028506, 2019.

21. Yazdi AS, Drexler SK and Tschopp J: The role of the inflammasome in nonmyeloid cells. J Clin Immunol 30: 623-627, 2010.

22. Lei-Leston AC, Murphy AG and Maloy KJ: Epithelial cell inflammasomes in intestinal immunity and inflammation. Front Immunol 8: 1168, 2017.

23. Dihlmann S, Erhart P, Mehrabi A, Nickkholgh A, Lasitschka F, Bockler D and Hakimi M: Increased expression and activation of absent in melanoma 2 inflammasome components in lymphocytic infiltrates of abdominal aortic aneurysms. Mol Med 20: 230-237, 2014

24. Barnard GA: A new test for $2 \times 2$ tables. Nature 156: 177, 1945.

25. Erhart P, Grond-Ginsbach C, Hakimi M, Lasitschka F, Dihlmann S, Bockler D and Hyhlik-Dürr A: Finite element analysis of abdominal aortic aneurysms: Predicted rupture risk correlates with aortic wall histology in individual patients. J Endovasc Ther 21: 556-564, 2014.

26. Erhart P, Schiele S, Ginsbach P, Grond-Ginsbach C, Hakimi M, Bockler D, Lorenzo-Bermejo J and Dihlmann S: Gene expression profiling in abdominal aortic aneurysms after finite element rupture risk assessment. J Endovasc Ther 24: 861-869, 2017.

27. Li H, Bai S, Ao Q, Wang X, Tian X, Li X, Tong H, Hou W and Fan J: Modulation of Immune-Inflammatory responses in abdominal aortic aneurysm: Emerging molecular targets. J Immunol Res 2018: 7213760, 2018.

28. Li X, Deroide N and Mallat Z: The role of the inflammasome in cardiovascular diseases. J Mol Med (Berl) 92: 307-319, 2014.

29. Usui F, Shirasuna K, Kimura H, Tatsumi K, Kawashima A, Karasawa T, Yoshimura K, Aoki H, Tsutsui H, Noda T, et al: Inflammasome activation by mitochondrial oxidative stress in macrophages leads to the development of angiotensin II-induced aortic aneurysm. Arterioscler Thromb Vasc Biol 35: 127-136, 2015.

30. Bobryshev YV and Lord RS: Vascular-associated lymphoid tissue (VALT) involvement in aortic aneurysm. Atherosclerosis 154: 15-21, 2001. 
31. Ocana E, Bohorquez JC, Perez-Requena J, Brieva JA and Rodriguez C: Characterisation of $\mathrm{T}$ and $\mathrm{B}$ lymphocytes infiltrating abdominal aortic aneurysms. Atherosclerosis 170: 39-48, 2003.

32. Johnston WF, Salmon M, Su G, Lu G, Stone ML, Zhao Y, Owens GK, Upchurch GR Jr and Ailawadi G: Genetic and pharmacologic disruption of interleukin-1 $\beta$ signaling inhibits experimental aortic aneurysm formation. Arterioscler Thromb Vasc Biol 33: 294-304, 2013

33. Isoda K, Akita K, Kitamura K, Sato-Okabayashi Y, Kadoguchi T, Isobe S, Ohtomo F, Sano M, Shimada K, Iwakura Y and Daida H: Inhibition of interleukin-1 suppresses angiotensin II-induced aortic inflammation and aneurysm formation. Int J Cardiol 270: 221-227, 2018.

34. Ridker PM, Thuren T, Zalewski A and Libby P: Interleukin-1 $\beta$ inhibition and the prevention of recurrent cardiovascular events: Rationale and design of the canakinumab anti-inflammatory thrombosis outcomes study (CANTOS). Am Heart J 162: 597-605, 2011
35. Shah SR, Abbasi Z, Fatima M, Ochani RK, Shahnawaz W, Asim Khan M and Shah SA: Canakinumab and cardiovascular outcomes: Results of the CANTOS trial. J Community Hosp Intern Med Perspect 8: 21-22, 2018.

36. Weber $C$ and von Hundelshausen P: CANTOS trial validates the inflammatory pathogenesis of Atherosclerosis: Setting the stage for a new chapter in therapeutic targeting. Circ Res 121: 1119-1121, 2017.

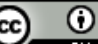

This work is licensed under a Creative Commons Attribution 4.0 International (CC BY 4.0) License. 